\title{
Violencia sutil y celos en una relación de pareja en estudiantes de una universidad pública de Lima Este
}

\author{
Subtle violence and jealousy in a relationship between students in a public university \\ in Lima East
}

\author{
Cynthia Peña Tasayco', Deyli Ticlla Sánchez², Cristian Adriano Rengifo³
}

\begin{abstract}
RESUMEN
Objetivo: Determinar la relación significativa entre violencia sutil y celos en una relación de pareja en estudiantes de una universidad pública de Lima Este. Metodología: Se utilizó el diseño no experimental de corte transversal y de alcance correlacional. Asimismo, se manejó un muestreo no probabilístico intencional, conformado por 242 jóvenes de ambos sexos con edades de 18 a 27 años. Los instrumentos utilizados para la recolección de datos fueron la Escala de Violencia Encubierta (EVE) de Lascorz (2015) y el Inventario Multidimensional de Celos (Diaz-Loving et al. 1989). Resultados: Evidenciaron que existe una asociación altamente significativa entre violencia sutil y celos $(P<.001)$. Se encontró que el $75.6 \%$ de los participantes manifestó haber utilizado en alguna ocasión en su vida violencia sutil con sus parejas; de la misma forma el $66.1 \%$ de los estudiantes es propensa a demostrar comportamientos inadecuados al percibir o malinterpretar la interacción de su pareja con sus pares. Conclusiones: Se comprobó que existe una asociación altamente significativa entre violencia sutil y celos, demostrando que ambas variables coexisten de forma activa en una relación de pareja.
\end{abstract}

Palabras clave: Violencia sutil; celos; parejas.

\begin{abstract}
Objective: determine the significant relationship between subtle violence and jealousy in a couple relationship in students of a public university in East Lima. Methodology: The non-experimental crosssectional design with correlational scope was used. Likewise, an intentional non-probabilistic sampling was conducted, conformed 242 young people of both sexes with ages between 18 and 27 years. The instruments used for data collection were the Covert Violence Scale (EVE) of (Lascorz, 2015) and the Inventory Multidimensional of Jealousy (Diaz-Loving et al., 1989). Results: They showed that there is a highly significant association between subtle violence and jealousy $(P<0.001)$. In which he found that $75.6 \%$ of the participants stated that they had used subtle violence with their partners at some time in their lives; In the same way, $66.1 \%$ of students are prone to demonstrate inappropriate behaviors when perceiving or misinterpreting their partner's interaction with their peers. Conclusions: It was found that there is a highly significant association between subtle violence and jealousy, demonstrating that both variables are related to each other within a couple relationship.
\end{abstract}

Keywords: Subtle violence; Jealousy; Couples.

${ }^{1}$ Lic. Psicología, Universidad Peruana Unión, Lima, Perú

${ }^{2}$ Lic. Psicología, Universidad Peruana Unión, Lima, Perú

${ }^{3}$ Docente EP Psicología, Universidad Peruana Unión, Lima, Perú 


\section{INTRODUCCIÓN}

A nivel mundial la violencia en sus diversas manifestaciones es identificada como un problema de salud pública. La Organización Mundial de la Salud (OMS, 2017) afirma que 1 de cada 3 mujeres es víctima de violencia física o sexual; asimismo, de los asesinatos pasionales que se cometen, un $38 \%$ es de mujeres y un $6 \%$, de varones.

La violencia en las relaciones de noviazgo juvenil fluctúa entre el 6 y $86 \%$; ya que depende en gran medida del tipo de violencia ejercida (Fernández y Fuertes, 2010), de modo que entre un 30 a $40 \%$ de las personas jóvenes sufre algún tipo de violencia física por parte de sus parejas (Muñoz, Graña, Leary y González, 2007). Asimismo, algunos estudios muestran que entre un $70 \%$ y un $80 \%$ de las mujeres que reciben estos maltratos, no se aprecian como víctimas (García et al., 2013).

En el Perú, según el Ministerio de la Mujer y Poblaciones Vulnerables, (MIMPV, 2015) de acuerdo con el Programa Nacional Contra la Violencia Familiar y Sexual (PNCVFS) se menciona que se han atendido 53675 denuncias por violencia familiar en Lima y provincias en los Centros de Emergencia Mujer (CEM), siendo un $85 \%$ de mujeres; además, un $62 \%$ de estos casos fue registrado por la población adulta de 18 a 59 años. El Instituto Nacional de Estadística e Informática (INEI, 2013) afirma que el tipo de violencia más común es el psicológico y/o verbal, expresado en un $67.5 \%$ de los casos.

Esta situación alarmante propicia que los investigadores cuestionen el porqué del fenómeno, buscando respuestas en el origen del problema como lo es la violencia sutil.

San Pablo (2017) menciona que la violencia es el uso de la fuerza para conseguir un fin; sin embargo, la violencia sutil es el uso de conductas verbales y no verbales que pueden aparecer en situaciones amorosas, graciosas y de cariño (Marshall, 1999) esto afecta más a la mujer, dañando su imagen y la forma de percibir la relación ya que el hombre ejerce presión mediante micro abusos y micro violencias a fin de imponer y mantener un dominio total sobre esta, (Bonino, 2011). Además, dicha conducta tiene menor probabilidad de ser detectado a tiempo y denunciado, ya que se asocia a la gravedad y al aumento en la severidad del daño psicológico (Fariña, Arce, Vilariño y Novo, 2014).
La violencia sutil o invisible es considerada como un suceso normal por lo cual se fomenta una complicidad y las personas no lo identifican fácilmente (San Pablo, 2017).

Por su parte, Blázquez, Moreno y GarcíaBaamonde (2010) consideran que son maniobras agresivas que no se basan en la fuerza física por lo cual son difíciles de identificar, creando en la víctima sentimientos de desvalorización que anula la autoestima y genera una indefensión aprendida. Asimismo, las críticas recurrentes, la agresión verbal, actos de aislamientos y dominación hacia la pareja son acciones que causan temor y un pobre autoconcepto (Lascorz, 2015).

Frente a esta problemática es necesario poder identificar los factores determinantes que influyen en la violencia, según Ocampo y Amar (2011) existen aspectos de la personalidad de cada individuo, las pautas de crianza y la interacción social, familiar y cultural que influyen en generar violencia en una relación de pareja. Siendo que en esta situación se encuentran comprometidos los sentimientos y muchas otras decisiones, lo vuelve un ambiente idóneo para que afloren los celos. Para Canto, Garcia, y Gomez (2009) cuando las expresiones de celos son de manera constante y malintencionada funcionan como causas desgastantes de la relación.

Los celos son reacciones afectivas o sentimientos que reflejan conductas que se encuentran ligadas al control de la situación (Diaz y Rivera, 2010). Asimismo, Martínez-León et al. (2013) refieren que los celos son emociones que nacen como resultado de un desmedido interés por conservar de manera exclusiva a una persona en una relación sentimental, basándose en una supuesta infidelidad de la persona amada que, solo en ocasiones, resulta ser real. Se puede considerar un nivel de celos normal cuando la persona muestra preocupación gratitud e interés en la pareja, pudiendo ser una expresión de amor acostumbrado. Sin embargo el surgimiento de celos intensos muestra asiduamente inseguridad, miedo, sentimientos de pertenencia sobre la pareja e intranquilidad por perderla ante un posible rival, resultando difícil establecer un margen entre los celos normales y los celos patológicos; estos últimos constituyen un problema de gran preocupación ya que muestra pensamientos, emociones y actitudes irracionales sobre el adulterio de la pareja, provocando alteraciones emocionales 
que conducen a la persona a desdoblar diversas conductas compulsivas con el único propósito de controlar a la otra persona.

En la actualidad, no se encuentran investigaciones que tengan relación significativa de violencia sutil y celos; sin embargo, existen estudios de celos, estilos de apego y el amor adictivo. Así, Retana y Sánchez (2008) muestran que cada estilo de apego manifiesta diversas emociones y conductas destructivas que afectan la relación de pareja.

Blazquez, Moreno y García-Baamonde (2011) afirman que los jóvenes de 17 o 18 años muestran en sus relaciones amorosas comportamientos destructivos hacia su pareja como humillaciones, amenazas, celos entre otros. Para Lewis y Fremouw, el periodo en el cual existe mayor riesgo de violencia es de 20 a 24 años, esto indica que a edades tempranas los jóvenes muestran el uso de la violencia psicológica dentro de sus relaciones amorosas (citado por Blazquez et al., 2011). De esta manera la población considerada para la investigación es de jóvenes de 18 a 27 años, ya que se encuentran en una etapa en la que se establecen relaciones de pareja más o menos estables. Asimismo, se vio conveniente optar por una universidad pública de Lima-Este en la cual exista una diversidad cultural y se ajuste a la naturaleza de la investigación.

En suma, todo lo anterior apunta al propósito central de esta investigación que es examinar la relación entre violencia sutil y celos en estudiantes de una universidad pública de LimaEste.

\section{METODOLOGÍA}

La presente investigación tiene un diseño no experimental de corte transversal y de tipo correlacional, porque no se manipularon las variables y los datos fueron recolectados y analizados en un solo momento. Además, este estudio es de alcance correlacional, debido a que se busca la relación entre violencia sutil y celos (Hernández, Fernández y Baptista, 2006).

\section{Muestra}

La muestra estuvo conformada por 242 estudiantes de ambos sexos de 18 a 27 años de edad, pertenecientes a las facultades de Agropecuaria y Nutrición y de Pedagogía y Cultura Física de una universidad pública. Fueron seleccionados a través de un muestreo no probabilístico intencional.

\section{Instrumento}

Los componentes de los instrumentos aplicados incluyeron una declaración de consentimiento informado, datos sociodemográficos relevantes y la Escala de Violencia Encubierta (EVE) y el Inventario Multidimensional de Celos.

\section{Escala de Violencia Encubierta (EVE)}

Elaborada por Bosch en el 2008, posteriormente fue adaptada a la población española en el 2015 por Lascorz denominándola Escala de Violencia Encubierta (EVE). Está constituida por cinco dimensiones: Inseguridad emocional, evasión de responsabilidad, coerción, control e infravaloración. Compuesta por 24 ítems de 2 preguntas, con una escala de respuesta tipo Likert que van desde "nunca" hasta "de forma habitual" con una puntuación de 0 a 3 respectivamente. Para hallar la validez de contenido según los coeficientes de validez de $\mathrm{V}$ deAiken, en la presente investigación, se utilizó el criterio de 5 jueces expertos conformado por tres psicólogos clínicos especialistas en diagnóstico, una psicóloga clínica comunitaria especializada en trabajar con mujeres víctimas de violencia y un psicólogo forense especializado en medicina legal y ciencias forenses. En la evaluación, los ítems 6.1 y 6.2 presentaron observaciones por tres jueces en cuanto a la congruencia, contexto y constructo, haciéndose las correcciones y la adaptación respectiva. Para ver la fiabilidad se utilizó el cociente alfa de Cronbach, obteniendo un puntaje de .94. Finalmente, para sacar la validez del constructo se utilizó el análisis de $r$ de Pearson sub test - test evidenciándose una validez adecuada $(p<.000)$.

\section{Inventario Multidimensional de Celos}

Creado y elaborado por Diaz - Loving et al. (1989), adaptado en el Perú por Bautista (2017) el cual evalúa el sentimiento de incomodidad y desconcierto que percibe un sujeto cuando percibe distinciones de una pareja hacia otra persona. Presenta 40 ítems de tipo Likert puntuados con 5 alternativas de respuestas (completamente en desacuerdo, en desacuerdo, ni en acuerdo ni en desacuerdo, de acuerdo y 
completamente de acuerdo). codificando 1 para la primera elección y 5 para la última opción. Dicho inventario consta de cinco dimensiones: Emocional/dolor, enojo, egoísmo, confianza e intriga. Referente a su administración se puede aplicar de forma individual, colectiva y autoaplicar, el tiempo aproximado para la resolución es de 15 a 20 minutos. La edad de las personas en las que se puede aplicar dicho instrumento es desde los 17 años en adelante que hayan mantenido o tengan una relación de pareja. La fiabilidad del inventario fue realizada con el coeficiente de Alpha de Cronbach, siendo superiores a .70 en todos los factores; en el factor emocional/ dolor se adquirió un .78; en el factor enojo se obtuvo un .79; en el factor egoísmo se logró un .78; en el factor confianza se adquirió un $.81 \mathrm{y}$, finalmente, en el factor intriga se alcanzó un .75.

\section{RESULTADOS}

En la tabla 1 se observa las características sociodemográficas de los participantes: Respecto al género, el $41.7 \%$ es de género masculino y el $58 \%$, femenino. Respecto a la edad, existe un $72.3 \%$ que tiene entre 18 a 22 años y un $27.7 \%$ entre 23 a 27 años. Asimismo, un $84.7 \%$ es de la costa, mientras que un $0.8 \%$ es de la selva. Además, el $66.5 \%$ es de la Facultad de Pedagogía y Cultura Física y el $33.5 \%$ pertenece a la Facultad de Agropecuaria y Nutrición.

Tabla 1

Datos sociodemográficos de los participantes.

\begin{tabular}{lll} 
Variable & $\mathrm{N}$ & $\%$ \\
Sexo & & \\
Masculino & 101 & $41.7 \%$ \\
Femenino & 141 & $58 \%$ \\
Edad & & \\
18 a 22 años & 175 & $72.3 \%$ \\
23 a 27 años & 67 & $27.7 \%$ \\
Procedencia & & \\
Costa & 205 & $84.7 \%$ \\
Sierra & 35 & $14.5 \%$ \\
Selva & 2 & $0.8 \%$ \\
Facultad & & \\
Agropecuaria y nutrición & 81 & $33.5 \%$ \\
Pedagogía y cultura física & 161 & $66.5 \%$ \\
\hline
\end{tabular}

En la tabla 2 se observa que el $75.6 \%$ de los participantes mencionó utilizar rara vez la violencia sutil en su relación, asimismo un $7.4 \%$ se identificó como víctima y un $11.6 \%$ de los participantes indicó haber alternado el rol de víctimas y victimarios. Finalmente, se aprecia que un $5.4 \%$ reconoció utilizar la violencia sutil contra su pareja. 
Tabla 2

Nivel de violencia sutil.

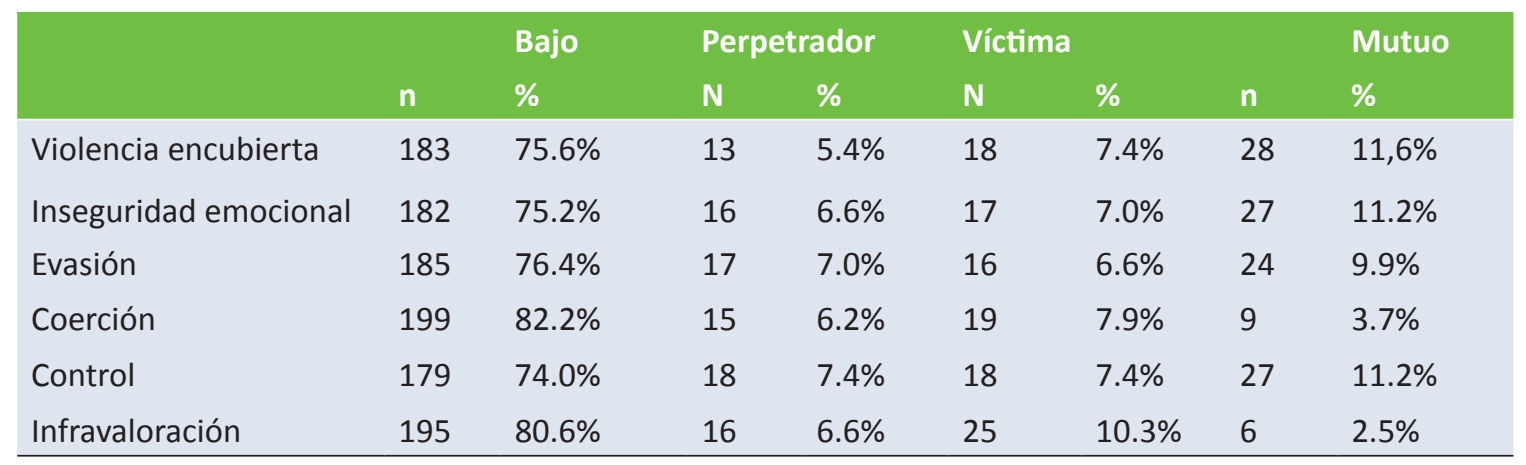

En relación al nivel de celos, la tabla 3 muestra que el $9.5 \%$ de los participantes posee altos niveles de celos, indicando que suele utilizar comportamientos negativos al percibir o malinterpretar la interacción de su pareja con sus pares. Por su parte, se observa que el $66.1 \%$ presenta niveles moderados de inseguridad y celos, mientras que solo un $24 \%$ presenta bajo nivel de celos.

Tabla 3

Nivel de celos.

\begin{tabular}{|c|c|c|c|c|c|c|}
\hline & & Bajo & & dio & & Alto \\
\hline & $\mathbf{n}$ & $\%$ & $\mathbf{N}$ & $\%$ & $n$ & $\%$ \\
\hline Celos & 59 & $24 \%$ & 160 & $66.1 \%$ & 23 & $9.5 \%$ \\
\hline Emocional/Dolor & 60 & $24.8 \%$ & 131 & $54.1 \%$ & 51 & $21.1 \%$ \\
\hline Enojo & 60 & $24.8 \%$ & 127 & $52.5 \%$ & 55 & $22.7 \%$ \\
\hline Egoísmo & 65 & $28.9 \%$ & 117 & $48.3 \%$ & 60 & $24.8 \%$ \\
\hline Confianza & 60 & $24.8 \%$ & 128 & $52.9 \%$ & 54 & $22.3 \%$ \\
\hline Intriga & 64 & $26.4 \%$ & 123 & $50.8 \%$ & 55 & $22.7 \%$ \\
\hline
\end{tabular}

En cuanto a la relación entre violencia sutil y celos, la tabla 4 muestra que existe una asociación altamente significativa $(P<0.001)$. De manera similar ocurre con las dimensiones y la variable celos, ya que se aprecia una alta asociación en cada una de ellas.

Tabla 4

Distribución probabilística de chi-cuadrado de Pearson.

\begin{tabular}{llll} 
& $\mathrm{x}^{2}$ & $\mathrm{Glz}$ & $\mathrm{P}$ \\
Violencia sutil y celos & $64.949^{\mathrm{a}}$ & 6 & .000 \\
Emocional/Dolor & $86.598^{\mathrm{a}}$ & 6 & .000 \\
Enojo & $70.437^{\mathrm{a}}$ & 6 & .000 \\
Egoísmo & $63.515^{\mathrm{a}}$ & 6 & .000 \\
Confianza & $30.372^{\mathrm{a}}$ & 6 & .000 \\
Intriga & $86.598^{\mathrm{a}}$ & 6 & .000 \\
\hline
\end{tabular}


En la figura 1 se aprecia que los estudiantes que son solo víctimas de violencia sutil presentan una categoría baja en celos, mientras que los que poseen un nivel bajo en violencia sutil muestran una categoría media en celos, además las personas que han obtenido un nivel mutuo en violencia sutil (agresor y víctima) presentan una categoría alta en celos.

Figura 1

Cuadro simétrico de normalización de violencia sutil y celos.

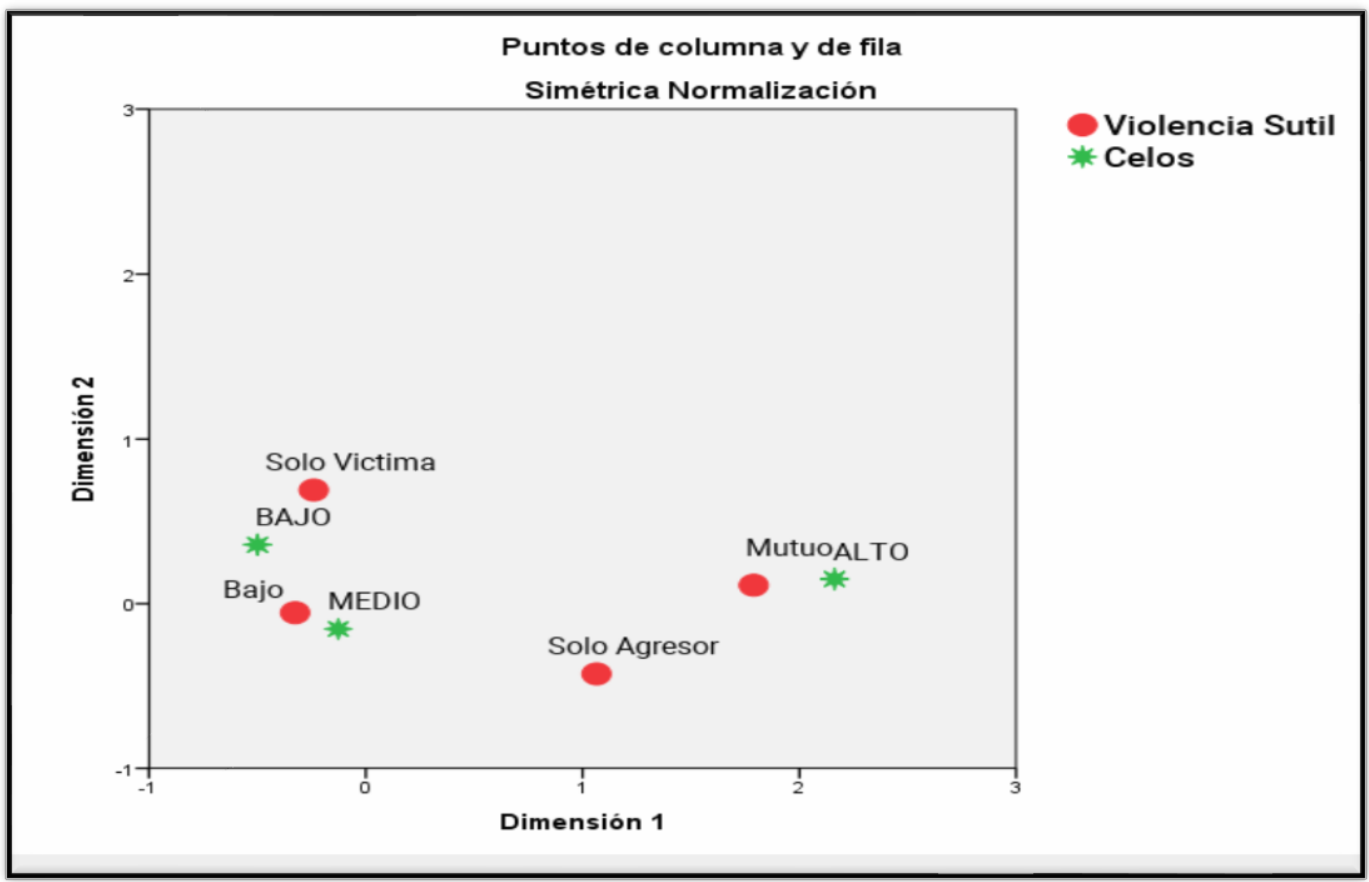

\section{DISCUSIÓN}

Es evidente que en la actualidad la violencia se ha incrementado significativamente, llegando a alcanzar altos índices tanto en nuestro territorio nacional como en el ámbito internacional. Una de las causas principales de esta problemática son los celos que, usualmente, se manifiestan por la desconfianza y la inseguridad emocional dentro de una relación de pareja.

En este sentido, los resultados encontrados en la investigación evidencian que existe una asociación altamente significativa entre violencia sutil y celos $(x 2=64.949 a ; p<.01)$ es decir que, tanto la violencia encubierta como los celos, pueden estar presentes de forma activa en una relación de pareja. Estos sucesos no son detectados fácilmente por las víctimas ya que no se manifiesta como violencia física sino como algo "cotidiano" y "normal" dentro de la sociedad, llegando incluso al extremo de que el perpetrador tome las decisiones que le corresponden a la víctima, por lo que esta violencia sutil, con el paso del tiempo, se puede convertir en violencia directa. Este resultado es respaldado por los datos estadísticos del Ministerio de la Mujer y Poblaciones vulnerables quien menciona que en el año 2018 se han reportado 113,727 denuncias por violencia de género, de los cuales el $85.1 \%$ de víctimas corresponde a mujeres y el $14.9 \%$, a varones. En cuanto a las motivaciones, el $67 \%$ de estas agresiones se dio por celos, por lo que el $21 \%$ decidió separarse debido a que la pareja invadía su privacidad, tomaba decisiones $y$, sobre todo, controlaba las actividades que realizaba (Infobae, 02 de mayo del 2018). Sobre la base de estos resultados, los investigadores Martinez-León, et al. (2013) afirman que los celos dentro de una relación de pareja conllevan al maltrato psicológico, físico y casos más trágicos como la muerte.

Del mismo modo, se encontró una asociación altamente significativa entre violencia sutil $y$ la dimensión Emocional/dolor (x2 = 86.598a; 
$p<.01)$ demostrando que estas emociones pueden provocar reacciones impulsivas; esto es respaldado por Ochoa (1998) y Lascorz (2015) quienes mencionan que estas expresiones y emociones salen a relucir ante una posible pérdida de la pareja o una aparente infidelidad, siendo expresada mediante críticas recurrentes, actos de aislamiento de su entorno social y dominación, causando temor y un pobre autoconcepto en la pareja. Como se aprecia en la tabla 7, la figura masculina muestra un mayor sentido de posesión y celos con su pareja debido a los prejuicios de la sociedad ya que todavía se mantiene el estereotipo de que el varón imperativamente debe ser el "macho" $y$, en ese contexto debe mantener una imagen, imponiendo sus decisiones y teniendo el control de la pareja, mediante la fuerza y dominación (Fernández Rius, 2008).

Asimismo, se halló una asociación altamente significativa entre violencia sutil y la dimensión Enojo (x2 = 70.437a; $p<.01$ ), lo que indica que los jóvenes manifiestan indicadores de inseguridad emocional, cólera y disgusto hacia la pareja por diversos motivos como la poca atención brindada, el temor ante una posible pérdida por causa de una tercera persona o recuerdos de relaciones anteriores, según Díaz, Rivera y Flores (1989) llegando a reprochar a la pareja de manera hostil, trayendo al presente sucesos pasados, realizando comentarios maliciosos, todo esto con el único afán de conseguir que la pareja se adapte a sus exigencias (Bonino, 2011; Lascorz, 2015).

De la misma manera, existe una asociación altamente significativa entre violencia sutil y egoísmo (x2 = 63.515a; $p$ < .01); pues, dentro de una relación de pareja, ambos se evidencian de forma paralela ya que una de las partes puede considerar que su pareja es su propiedad, limitando su interacción con las personas que le rodean. Esto concuerda con Bonino (2011), quien menciona que las personas que hacen uso de violencia sutil en una relación de pareja intentan limitar su libertad restringiendo su capacidad de decisión. Del mismo modo, Montes-Berges (2008) indican que los celos románticos están relacionados con el uso de tácticas manipuladoras con la finalidad de que la pareja no interactúe con sus pares.

Por otro lado, se encontró que existe una asociación altamente significativa entre violencia sutil y la dimensión confianza con un cociente Chi-cuadrado de Spearman ( $\mathrm{x} 2=$ $30.372 a ; p<.01$ ), evidenciando que la violencia sutil interactúa activamente con la desconfianza en una relación de pareja, corroborado con la teoría biopsicosociocultural - componente biológico, en la que Shaver, Hazan y Bradshaw (1987) revelan que la niñez influye en el proceso socioemocional de los individuos como causa del desarrollo, entre estos se encuentra el apego ansioso - ambivalente y el apego de evitación, ya que en la niñez el individuo no se sintió seguro de la incondicionalidad del cuidador llevándolo a que necesite constantemente aprobación. Estos niños se encuentran preocupados de la relación con su cuidador ya que en algunas ocasiones estos muestran respuestas afectuosas, en otras, enfado o los ignoran. Por ello, los niños presentan temor a ser abandonados por lo que vigilan constantemente al cuidador, asimismo, presentan dificultad para adaptarse y ansiedad emocional porloqueenlaetapaadultamanifiestan inseguridad emocional, conductas impulsivas, distintas relaciones amorosas y desconfianza continua al mantener una relación de pareja. Esto lo refuerza Alayo (2017) quien reporta que estas conductas se deben a la presencia del temor a ser engañados, desconfiando en todo momento de las palabras y las acciones de su pareja. Asimismo, Ochoa (1998) refiere que las personas celosas desconfían de su pareja, por lo que manifiestan conductas negativas como inspeccionar las pertenencias del otro, seguirlo constantemente o preguntarle dónde y con quién ha estado. Todo esto, a su vez, genera una baja autoestima en la víctima (Mayor, 2013) y (Acosta y Vidarte, 2015).

Finalmente, se encontró que existe una asociación altamente significativa entre la violencia sutil y la dimensión Intriga ( $\mathrm{x} 2=86.598 \mathrm{a}$; $p<.01)$ entendiendo que en la relación de pareja la persona celosa se encuentra perturbada con deseos de saber qué actividades realiza su pareja cuando no está presente, además de sospechar que su pareja está confabulando algo contra él (Bautista, 2017). Como resultado de estas percepciones, las personas celosas tratan de buscar respuestas y al no encontrarlas muestran una reacción no favorable haciendo uso de reclamos sin sentido hasta llegar a los gritos e insultos con el fin de dominar la relación y estar al tanto de lo que hace su pareja para sentirse seguro(a) y mitigar sus pensamientos paranoicos (Lascorz, 2015 y Alayo Castillo, 2017). 


\section{Declaración de financiamiento y de conflictos de interés:}

El estudio fue financiado por los autores, quienes declaran no tener conflictos de interés.

\section{Correspondencia}

Cynthia Peña Tasayco

Correo electrónico:

cynthiatasayco@upeu.edu.pe

\author{
Deyli Ticlla Sánchez \\ Correo electrónico: \\ deyliticlla@upeu.edu.pe \\ Cristian Adriano Rengifo \\ Correo electrónico: \\ cristianadriano@upeu.edu.pe
}

\section{REFERENCIAS BIBLIOGRÁFICAS}

Acosta, A.y Vidarte, D. (2015). Celos en la relación de pareja en estudiantes del primero al noveno ciclo de psicología de una universidad privada de la ciudad de Chiclayo 2015. (Tesis de grado, Universidad Privada Juan Mejía Baca). Recuperado d e http://repositorio.umb.edu.pe/bitstream/UMB/97/1/ Acosta Segura \%26 Vidarte Cabrera.pdf

Alayo Castillo, F. (2017). Celos y Variables Sociodemográficas en Jóvenes Universitarios de la Ciudad de Trujillo. (Tesis de grado, Universidad César Vallejo). Recuperado d e http://repositorio.ucv.edu.pe/bitstream/handle/ UCV/644/alayo_cf.pdf?sequence=1\&isAllowed=y

Bautista, E. (2017). Propiedades Psicométricas del inventario multidimensional de celos en adultos jóvenes del distrito de la esperanza (Tesis de grado, Universidad César Vallejo, $\quad$ Perú ). Recuperado de http://repositorio.ucv.edu.pe/ handle/UCV/654

Bonino Menedez, L. (2011). Micromachismos: La violencia invisible en la pareja. Jornadas Del a Federación de Sociedades Españolas de Terapia Familiar, 1, 1-19. Recuperado de: https:// primeravocal.org/micromachismos-la-violenciainvisible-en-la-pareja-de-luis-bonino-mendez/

Blázquez, M., Moreno, J., y García, M. (2010). Revisión teórica del maltrato psicológico en la violencia conyugal. Psicología y Salud, 20(34), 65-75. Recuperado de https://www.uv.mx/psicysalud/ psicysalud-20-1/20-1/Macarena-Blazquez-Alonso. pdf
Blázquez, M., Moreno, J.,y García-Baamonde, E. (2011). Desarrollo de la violencia psicológica durante el noviazgo en parejas de jóvenes universitarios/ as. Apuntes de Psicología, 29(3), 397-412. Recuperado de http://apuntesdepsicologia.es/ index.php/revista/article/view/238/240

Diaz Loving , R., y Rivera Aragon , S. (2010). Antología Psicosocial de la pareja: Clásicos y contemporáneos (Tesis de grado, Universidad Nacional Autónoma de México- Facultad de Psicología, México).

Díaz, R., Rivera, S., \&; Flores, M. (1989). Desarrollo y análisis psicométrico de una medida multidimensional de celos. Revista Mexicana de Psicología, 6 (2) 111- 119.

Fariña, F., Arce, R., Vilariño, M., y Novo, M. (2014). Assessment of the standard forensic procedure for the evolution of psychological injury in intimate - partner violence. The Spanish Journal of Psychology, 17 E32. doi: 10.1017/sjp.2014.30.

Fernández Rius, L. (2008). ¿violencia invisible o del éxtasis al dolor? Estudios Feministas, Florianópolis, 16(1) 288.

García, V., Fernández, A., Rodríguez, F., López, M., Mosteiro, M., y Lana, A. (2013). Violencia de género en estudiantes de enfermería durante sus relaciones de noviazgo. Atención primaria, 45(6), 290- 296. Recuperad de https://core.ac.uk/ download/pdf/81971077.pdf

Hernández Sampieri, R., Fernández Collado, C., \&amp; Baptista Lucio, P. (2006). Metodología de la investigación: Roberto Hernández Sampieri, Carlos Fernández Collado y Pilar Baptista Lucio (6a. ed. --.). México D.F.: McGraw-Hill.

Infobae. (2018). Cada dos días una mujer es asesinada en Perú por culpa del machismo. Recuperado de https://www.infobae.com/america/americalatina/2018/05/02/cada-dos-dias-una- mujer-esasesinada-en-peru-por-culpa-del-machismo/

Instituto Nacional de Estadística e Informática (2013). Violencia entre mujeres, niños y niñas. en INEI, Encuesta Demográfica y de Salud. Instituto Nacional de Estadista, 349- 393

Lascorz, A. (2015). Violencia encubierta en las relaciones de parejas jóvenes (Tesis Doctoral, Universidad de Castilla-La mancha, España). Recuperado de https://ruidera.uclm.es/xmlui/ handle/10578/8675

Martínez-León, N. C., García-rincón, L., BarretoCortes, D. S., Alfonso, A., Parra, A. y Duque, B. (2013). Características de los celos en un grupo de estudiantes universitarios de la ciudad de Bogotá. Cuadernos Hispanoamericanos de Psicología, 13(1), 
36-44. Recuperado de https://dialnet.unirioja. es/descarga/articulo/5493082.pdf

Marshall, L. (1999). Efectos del abuso psicológico sutil y manifiesto de los hombres en mujeres de bajos ingresos. Violencia y víctimas, 14(1), 69-88. Recuperado de https://psycnet.apa.org/ doi/10.1891/0886-6708.14.1.69

Mayor, V. (2013). Micromachismo en parejas jóvenes (Tesis de grado, Universidad de Sevilla, España). Recuperado de https://idus.us.es/xmlui/bitstream/ handle/11441/32781/TFG-52 Micromachismo en parejas jóvenes.pdf?sequence $=1$

Ministerio de la Mujer y Poblaciones Vulnerables (2015). Programa Nacional Contra la Violencia Familiar y Sexual (PNCVFS).

Montes Berges, B. (2008). Tácticas para la resolución de conflictos y celos románticos en relaciones íntimas: Adaptación y análisis de las escalas CTS2 y CR. Estudios de Psicología, 29(2), 221-234. Recuperado de https://doi. org/10.1174/021093908784485138

Ocampo, L., y Amar, J. (2011). Violencia en la pareja, las caras del fenómeno. Salud Uninorte, 27(1), 108-123. Recuperado de http://www.scielo.org. co/pdf/sun/v27n1/v27n1a11.pdf
Ochoa, S. G. (1998). Validez de Constructo y Confiabilidad del Inventario Multidimensional de Celos. (Tesis de Maestría, Universidad de Colima - Facultad de Psicología, México). Recuperado de http://bvirtual.ucol.mx/consultaxcategoria. php?categoria $=3 \&$ id $=5433$

Retana Franco, B., y Sánchez Aragón, R. (2008). El Papel de los Estilos de Apego y los Celos en la Asociación con el Amor Adictivo. Psicología Iberoamericana, 16(1), 15-22. Recuperado de https://www.redalyc.org/pdf/1339/133915922003. pdf

San Pablo Rubiño, I. (2017). Violencia Invisible (Tesis de grado, Universidad de Valladolid). Recuperado de http://uvadoc.uva.es/ bitstream/handle/10324/19479/TFG-G1882. pdf?sequence $=1$ \&isAllowed $=y$

Shaver, R., Haza, C., y Bradshaw, D. (1987). Love as attachment: The integration of three behavioral systems. New Haven, CT: Yale university Presst: The psychology of love. Recuperado de: https:// www.scirp.org/(S(czeh2tfqyw2orz553k1w0r45))/ reference/ReferencesPapers.aspx?Referencel $D=1930345$

Recibido: 20/02/19 Aceptado: 01/04/19 